Национальный медицинский исследовательский центр им. В.А. Алмазова, Санкт-Петербург

ОБОСНОВАНИЕ. Гестационный сахарный диабет (ГСД) является одним из самых частых нарушений углеводного обмена, встречающихся во время беременности. В связи с продолжающимся поиском оптимальных целевых значений гликемии во время беременности при ГСД актуальным является изучение влияния разного уровня гликемии на внутриутробное развитие плода и дальнейшие изменения показателей углеводного и липидного обмена у детей.

ЦЕЛЬ. Дать характеристику перинатального периода, физического развития и метаболического статуса детей, рожденных у женщин с ГСД и разным уровнем контроля углеводного обмена.

МЕтодЫ. Проведена оценка течения перинатального периода и антропометрических показателей у 300 детей, рожденных у женщин с ГСД и разным уровнем гликемии во время беременности. На протяжении двух лет у 141 ребенка оценены показатели физического развития, параметры углеводного и липидного обмена. У 33 детей в возрасте от 1 до 4 лет выполнено мониторирование гликемии глюкометром натощак и постпрандиально на протяжении 14 дней.

РЕзУЛЬТАТЫ. При гликемии женщин натощак менее 5,1 ммоль/л и 7,0 ммоль/л через 1 ч после еды антропометрические параметры детей при рождении не отличались от параметров группы контроля (р>0,05). Гликемия женщин выше данного уровня была ассоциирована с повышением частоты и риска возникновения показателя массы, отношения масса/длина тела и окружности головы «выше среднего» $(p<0,05)$. При динамическом контроле антропометрических параметров детей до 2 лет различий между группами сравнения получено не было (р>0,05). Наиболее выраженные изменения метаболических параметров представлены неонатальной гипогликемией у новорожденных группы ГСД (группа ГСД - 23\%, группа контроля - 3,5\%; p=0,000002), наименьший риск которой встречался у детей с наиболее низкими значениями гликемии женщин натощак и постпрандиально. При измерении уровня глюкозы натощак, инсулина, индекса инсулинорезистентности (HOMA-IR), триглицеридов и холестерина у детей в возрасте 6, 12 и 24 мес, а также при мониторировании гликемии натощак и постпрандиально на протяжении 14 дней у детей в возрасте до 4 лет значимых различий между группами сравнения получено не было (p>0,05).

ЗАКЛЮЧЕНИЕ. Наименьшие риски возникновения неонатальной гипогликемии и антропометрических отклонений при рождении ассоциированы с наиболее низкими значениями гликемии, соответствующими критериям Российских клинических рекомендаций.

КЛЮЧЕВЫЕ СЛОВА: гестачионный сахарный диабет; фетальное программирование; макросомия; гипергликемия; эпигенетика; INTERGROWTH-21 $1^{\text {st }}$

\title{
ASSESSMENT OF THE PHYSICAL DEVELOPMENT AND METABOLIC STATUS OF CHILDREN BORN TO WOMEN WITH GESTATIONAL DIABETES
}

\author{
๑ Alisa S. Deynega (Masel), Anastasiya S. Liskina, Salimet A. Valieva, Irina L. Nikitina
}

Almazov National Medical Research Centre, Saint-Petersburg, Russia

BACKGRAUND: Gestational diabetes mellitus (GDM) is one of the most common metabolic disorders found during pregnancy. Currently, it is relevant not only to search optimal target levels of glycemia during pregnancy, but also to study the effect of different glycemia levels on fetal development and further changes in glucose and lipid metabolism in children.

AIMS: To describe perinatal period, physical development and metabolic status of children born to women with GDM and different glucose levels during pregnancy.

MATERIALS AND METHODS: The perinatal period features and anthropometric parameters at birth were evaluated in 300 children born to women with GDM and different levels of glycemia during pregnancy. Over the course two years, 141 children have been evaluated for physical development parameters and glucose and lipid metabolism. Fasting and postprandial glycemia was measured with glucometer for 14 days in 33 children aged 1 to 4 years. 
RESULTS: The anthropometric parameters of children at birth did not differ from the parameters of the control group ( $>$ > 0.05) when during pregnancy fasting blood glucose was less than $5.1 \mathrm{mmol} / \mathrm{I}$ and $7.0 \mathrm{mmol} / \mathrm{I} 1 \mathrm{hour}$ after a meal. The glycemia in women above this level was associated with an increase of frequency and risk of a body mass index, body mass / length ratio and head circumference "above average" in children at birth $(p<0.05)$. With the dynamic control of anthropometric parameters up to 2 years, no differences between the comparison groups were obtained ( $p>0.05)$. The change in metabolic parameters was represented by neonatal hypoglycemia in children of GDM group (GDM group - 23\%, control group $-3.5 \%, p=0.000002$ ), the least risk of which occurred in group with the lowest fasting and postprandial glycemic values during pregnancy. Fasting glucose, and insulin levels, HOMA index, triglycerides and cholesterol, as well as monitoring fasting and postprandial glycemia for 14 days, were obtained no significant differences between the comparison groups of children $(p>0.05)$.

CONCLUSIONS: The lowest risks of neonatal hypoglycemia and anthropometric deviations at birth were associated with the lowest glycemia levels during pregnancy, which correspond to the criteria of the Russian clinical guidelines.

KEYWORDS: gestational diabetes; fetal programming; hyperglycemia; epigenetics, macrosomia, INTERGROWTH-2 $1^{\text {st }}$

B 1998 г. D.J. Barker была описана теория фетального программирования, в соответствии с которой генетическая предрасположенность, факторы внешней среды и патологические состояния со стороны женщины могут оказывать влияние на формирующийся плод и предопределять нарушения здоровья ребенка в будущем [1]. Гипергликемия (ед ч) во время беременности является одним из наиболее часто встречающихся эпигенетических факторов. В настоящее время каждая шестая беременность сопровождается синдромом гипергликемии, который в $84 \%$ случаев обусловлен развитием гестационного сахарного диабета (ГСД) [2, 3]. В 2012 г. в соответствии с российским национальным консенсусом были приняты новые диагностические критерии ГСД в Российской Федерации и установлены целевые цифры гликемии в течение дня у беременных женщин [4]. Следует отметить, что целевые значения глюкозы крови натощак и через 1 ч после приема пищи в соответствии с протоколом ряда международных ассоциаций различаются [2-6]. В связи с этим актуальным является вопрос воздействия гликемии разного уровня при ГСД на развитие детей в дальнейшей жизни.

\section{ЦЕЛЬ}

Дать характеристику перинатального периода, физического развития и метаболического статуса детей, рожденных у женщин с ГСД и разным уровнем контроля углеводного обмена.

\section{МЕТОДЫ}

\section{Дизайн исследования}

Проведенное нами исследование было выполнено в три этапа: первая часть работы представляла собой обсервационное одноцентровое ретроспективное выборочное контролируемое исследование. Вторая и третья части являлись обсервационными одноцентровыми проспективными выборочными контролируемыми исследованиями.

\section{Критерии соответствия}

Критериями включения детей в основную группу исследованиями являлись: наличие подтвержденного ГСД у беременной женщины; рождение на 38-42-й неделе гестации с оценкой по шкале Апгар на 1-й минуте не менее 7 баллов и на 5-й минуте не менее 8 баллов. Критериями исключения являлись: наличие других подтвержденных прегестационных форм сахарного диабета у беременной женщины, рождение ранее 38-й и позднее 42-й недели гестации; оценка по шкале Апгар менее 7 баллов на 1-й минуте и менее 8 баллов на 5-й минуте; наличие хирургической или хронической соматической патологии у детей.

\section{Условия проведения}

Набор и обследование пациентов проводились на базе ФГБУ «Национальный медицинский исследовательский центр им. В.А. Алмазова» амбулаторно и в условиях стационара.

\section{Продолжительность исследования}

Исследование проводилось с мая 2017 по февраль 2020 г. Включение пациентов проводилось с момента подтверждения у беременных женщин ГСД в срок с 24-й по 28-ю неделю гестации путем проведения перорального глюкозотолерантного теста. Наблюдение за детьми, рожденными у женщин с ГСД, проводилось в течение 4 лет. Анализ данных был выполнен с марта 2019 г. по февраль 2020 г. на базе кафедры детских болезней ФГБУ «НМИЦ им. В.А. Алмазова».

\section{Описание медицинского вмешательства}

Данные об уровне глюкозы крови во время беременности были получены путем проведения регулярных амбулаторных приемов эндокринолога и контроля электронных дневников гликемии натощак и через 1 ч после приема пищи при самостоятельном измерении женщинами гликемии при помощи глюкометра. Данные о течении перинатального периода были получены при анализе карт медицинской информационной системы QMS, включающем соматические заболевания женщин, потенциально влияющие на здоровье плода; течение интранатального и раннего неонатального периода; антропометрические показатели новорожденных с расчетом стандартного отклонения (SDS - standart deviation score). В возрасте 6, 12 и 24 мес была выполнена антропометрия детей с расчетом SDS. В раннем неонатальном периоде, в 6, 12 и 24 мес у детей был определен уровень некоторых метаболических показателей крови. Методом многократных измерений глюкозы крови глюкометром на протяжении 2 нед наблюдения была проведена оценка гликемии натощак и постпрандиально у детей в возрасте от 1 до 4 лет. 


\section{Основной исход исследования}

Основной конечной точкой исследования были отклонения метаболических и антропометрических показателей детей в зависимости от наличия ГСД и гликемии женщин во время беременности.

Дополнительные исходы исследования

Связь между наличием ГСДу женщин и отклонениями течения перинатального периода.

\section{Анализ в подгруппах}

Изначально анализ был проведен в двух группах наблюдения в зависимости от наличия ГСД у женщин:

- группа ГСД - 186 женщин и детей;

- группа без ГСД (контроль) - 114 женщин и детей.

В соответствии с уровнем гликемии женщин при ГСД дети были разделены на четыре группы:

- группа 1 - дети, рожденные у женщин с гликемией натощак <5,1 ммоль/л и <7,0 ммоль/л через 1 ч после приема пищи;

- группа 2 - дети, рожденные у женщин с гликемией натощак $\leq 5,3$ ммоль/л и/или $\leq 7,8$ ммоль/л через 1 ч после приема пищи;

- группа 3 - дети, рожденные у женщин с гликемией натощак >5,3 ммоль/л и/или >7,8 ммоль/л через 1 ч после приема пищи;

- группа 4 - группа контроля - дети, рожденные у женщин без ГСД.

\section{Методы регистрации исходов}

На первом этапе были оценены особенности течения перинатального периода при помощи статистических программ. Показатели физического развития детей при рождении в соответствии с полом и сроком гестации с расчетом SDS были проанализированы по стандартам INTERGROWTH-21 ${ }^{\text {st }}$ (длина, масса, соотношение масса/ длина тела и окружность головы). Антропометрические параметры в пределах \pm 1 SDS оценивались как «средние», отклонения более и менее 1 SDS оценивались «выше среднего» и «ниже среднего». В возрасте 6, 12 и 24 мес выполнялась антропометрия с расчетом SDS (масса и длина тела) по стандартам Всемирной организации здравоохранения для детей в соответствии с полом и возрастом. Антропометрические параметры в пределах \pm 1 SDS оценивались как «средние», отклонения более и менее 1 SDS оценивались «выше среднего» и «ниже среднего». В возрасте 6, 12 и 24 мес определение уровня глюкозы плазмы натощак выполнено глюкозооксидазным методом при использовании анализатора Abbott Architect 8000 (референс 3,29-5,5 ммоль/л); уровень инсулина в сыворотке крови определен иммуноферментным методом на анализаторе Cobas e411 (референс 17,8-173,0 пмоль/л); показатели общего холестерина (референс 0,00-5,17 ммоль/л) и триглицеридов (референс 0,00-1,69 ммоль/л) определены иммуноферментным методом при использовании анализатора Cobas Integra 400. Индекс инсулинорезистентности (HOMA-IR) был определен по формуле: инсулин натощак (пмоль/л) $\times$ глюкоза натощак (ммоль/л)/155. Показатель HOMA-IR считали в пределах нормы при значении менее 3,2 [7]. У детей в возрасте от 1 года до 4 лет женщин групп разного контроля гликемии при ГСД на протяжении 14 дней был выполнен мониторинг гликемии с использованием экспресс-анализатора (глюкометр) портативного АсcuChek Performa натощак и постпрандиально в течение дня, с последующим внесением данных в дневник самоконтроля. Контроль выполнения исследования осуществлялся посредством телефонных звонков исследователей и очных визитов.

\section{Этическая экспертиза}

Протокол исследования одобрен локальным этическим комитетом ФГБУ «НМИЦ им. В.А. Алмазова», выписка из протокола №5 от 17.05.2017 г. Все участники были включены в исследование после добровольного подписания формы информированного согласия.

\section{Статистический анализ}

Статистический анализ проводился с использованием пакета Microsoft Office 2013 (15.0) и программ Epi Info (Epi Info 7.2.2.16, CDC, США) и Jasp (JASP Team 2019. JASP, Version 0.9.2, Нидерланды).

Для сравнения распределения качественных признаков был использован критерий $\chi 2$, для сравнения переменных в независимых выборках применялся непараметрический U-критерий Манна-Уитни, при сравнении нескольких выборок использовались ранговый дисперсионный анализ Краскела-Уоллиса и метод логистической регрессии. Критический уровень значимости при проверке статистических гипотез принимался равным 0,05 .

\section{РЕЗУЛЬТАТЫ}

\section{Объекты (участники) исследования}

На первом этапе объектом исследования явились электронные карты 300 женщин и 300 доношенных новорожденных. В группу ГСД вошли данные 186 женщин и детей, в группу контроля - 114 . В соответствии с уровнем гликемии женщин во время беременности были сформированы группы наблюдения и проанализированы электронные карты детей. В группу 1 были включены 50 электронных карт, в группу 2 - 90, в группу 3 46 и в группу 4 (контроль) - 114 карт.

На втором этапе в течение первых двух лет жизни был обследован 141 ребенок. В группу ГСД был включен 101 ребенок, В группу контроля - 40 детей.

В третьем этапе приняли участие 33 ребенка, из них к группе 1 относились 10 детей, группе 2 - 9 детей, группе 3 - 6 детей, группе $4-8$ детей.

\section{Основные результаты исследования \\ Результаты первого этапа исследования}

Первым антропометрическим показателем, который был проанализирован в зависимости от наличия ГСД у женщин, явился признак макросомии новорожденных (вес более 4 кг или более 90 перцентиля для пола и гестационного возраста), статистических различий в представленности признака получено не было. Далее была проведена оценка массы, длины тела и окружности головы при помощи стандартов INTERGROWTH-21 Достоверные различия были получены в отношении увеличения количества детей в группе ГСД с показателями массы, соотношения масса/длина тела и окружности 
Таблица 1. Сравнение антропометрических показателей новорожденных детей групп с/без гестационного сахарного диабета

\begin{tabular}{|c|c|c|c|c|}
\hline Показатель & $\begin{array}{c}\text { Группа с ГСД, } \\
\text { n=186 (\%) }\end{array}$ & $\begin{array}{c}\text { Группа без } \\
\text { ГСД, } n=114 \text { (\%) }\end{array}$ & $\mathbf{p}^{*}$ & ОШ \\
\hline Вес более 4 кг/более 90 перцентиля & $25(13,4)$ & $9(7,9)$ & 0,2 & 1,8 \\
\hline SDS массы тела от +1 до +2 («выше среднего») & $65(35,5)$ & $19(16,8)$ & 0,0009 & 2,6 \\
\hline SDS соотношения масса/длина тела от +1 до +2 («выше среднего») & $31(16,7)$ & $7(6,1)$ & 0,007 & 3,1 \\
\hline SDS окружности головы от +1 до +2 («выше среднего») & $99(52,7)$ & $39(35,1)$ & 0,001 & 2,2 \\
\hline
\end{tabular}

Примечание: *- для статистического анализа был использован критерий $\chi^{2}$ для качественных признаков. ОШ — отношение шансов.

Таблица 2. Анализ антропометрических показателей новорожденных в зависимости от гликемии женщин во время беременности

\begin{tabular}{lccccccc}
\hline \multicolumn{1}{c}{ Показатель } & $\begin{array}{c}\text { Группа 1, } \\
\mathbf{n = 5 0}(\%)\end{array}$ & $\mathbf{p}^{*}$ & $\begin{array}{c}\text { Группа 2, } \\
\mathbf{n = 9 0}(\%)\end{array}$ & $\mathbf{p}^{* *}$ & $\begin{array}{c}\text { Группа 3, } \\
\mathbf{n = 4 6}(\%)\end{array}$ & $\mathbf{p}^{* * * *}$ & $\begin{array}{c}\text { Грyпnа 4, } \\
\mathbf{n = 1 1 4}(\%)\end{array}$ \\
\hline $\begin{array}{l}\text { SDS массы тела от +1 до +2 } \\
\text { («выше среднего) }\end{array}$ & $14(28)$ & 0,1 & $30(34)$ & 0,004 & $21(45,6)$ & 0,001 & $19(16,7)$ \\
$\begin{array}{l}\text { SDS соотношения масса/длина тела от +1 до +2 } \\
\text { («выше среднего») }\end{array}$ & $3(6)$ & 0,9 & $17(18,9)$ & 0,007 & $11(24,4)$ & 0,002 & $7(6,1)$ \\
$\begin{array}{l}\text { SDS окружности головы от +1 до +2 } \\
\text { («выше среднего) }\end{array}$ & $19(38)$ & 0,6 & $51(56,6)$ & 0,001 & $29(63)$ & 0,003 & $39(33,9)$ \\
\hline
\end{tabular}

Примечания: для статистического анализа был использован критерий $x^{2}$ для качественных признаков и метод логистической регрессии. * - сравнение группы 1 и группы 4; ** — сравнение группы 2 и группы 4; *** — сравнение группы 3 и группы 4.

головы «выше среднего» (табл. 1), при этом длина тела новорожденных в двух обследованных группах не различалась $(p>0,05)$.

В дальнейшем был проведен вторичный анализ полученных данных для установления различий между отдельными группами обследуемых детей (табл. 2).

Частота встречаемости признака массы тела «выше среднего» у новорожденных в группе 1 и группе 4 статистически не различалась, в то время как в остальных группах были получены значимые различия.

Принадлежность новорожденных к группам 2 и 3 по сравнению с группой 4 повышала риск рождения

$\%$

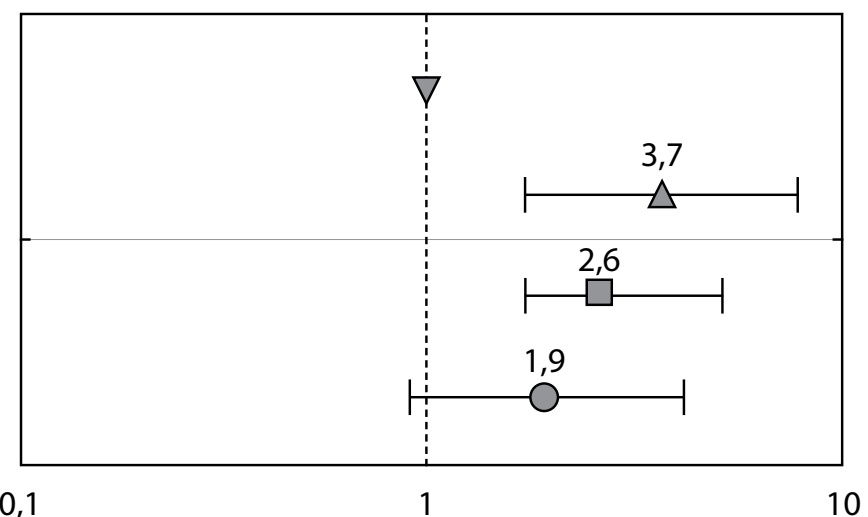

0,1

Отношение шансов детей с показателем массы тела «выше среднего», в то время как риски рождения ребенка с таким признаком в группах 1 и 4 не различались (рис. 1).

При оценке соотношения масса/длина тела в группах 2 и 3 установлено увеличение количества детей с данным показателем «выше среднего» по сравнению с группами 1 и 4.

В группах 2 и 3 по сравнению с группой 4 риск рождения ребенка с показателем соотношения масса/длина тела «выше среднего» был достоверно выше, в то время как при сравнении групп 1 и 4 данной тенденции не отмечалось (рис. 2).

$\%$

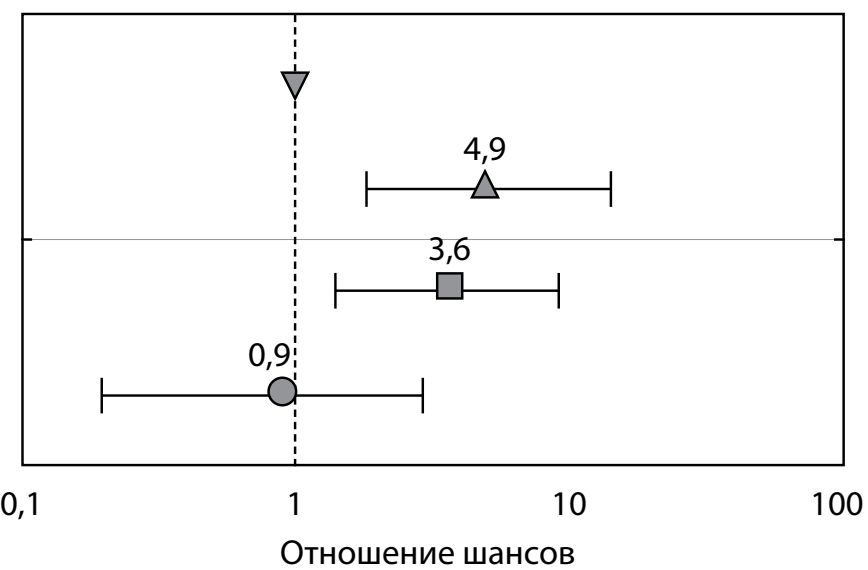

Группа $1 \square$ группа $2 \triangle$ Группа $3 \nabla$ группа 4
Рисунок 1. Риск рождения ребенка с показателем массы тела «выше среднего» (от +1 до +2 SDS) в зависимости от гликемии женщин во время беременности (статистический анализ проведен с использованием метода логистической регрессии).
Рисунок 2. Риск рождения ребенка с показателем соотношения масса/длина тела «выше среднего» (от +1 до +2 SDS) в зависимости от гликемии женщин во время беременности (статистический анализ проведен с использованием метода логистической регрессии). 
$\%$

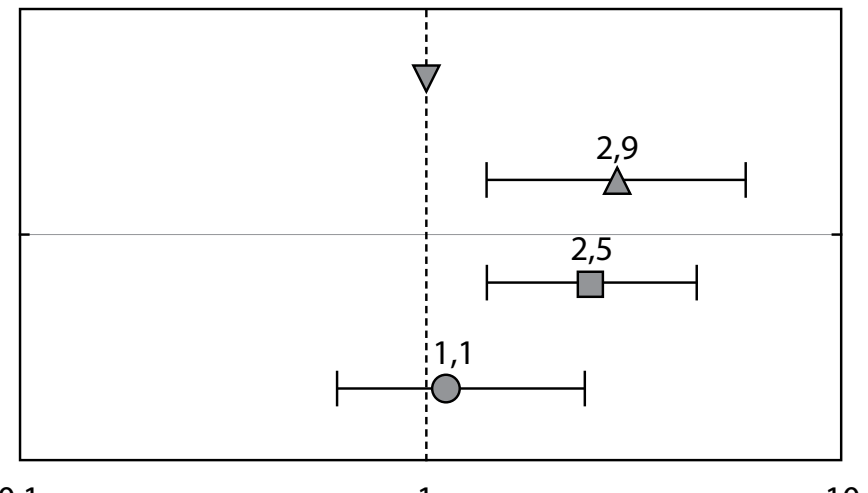

0,1

1

Отношение шансов

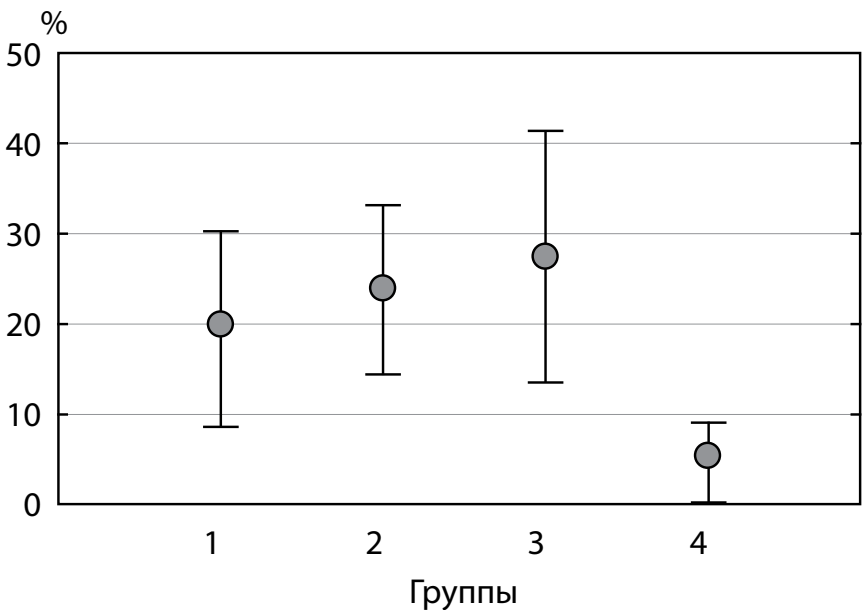

Рисунок 4. Встречаемость неонатальной гипогликемии в зависимости от гликемии женщин во время беременности (статистический анализ качественных значений проведен с использованием критерия $X^{2}$, $\mathrm{p}=0,000002)$.

женщин во время беременности. При оценке липидного (холестерин, триглицериды) и углеводного обмена (глюкоза натощак, инсулин, индекс HOMA-IR) не было выявлено значимых различий на протяжении всего периода наблюдения ( $p>0,05)$.

\section{Результаты третьего этапа исследования}

Стремление оценить углеводный обмен у детей в более старшем возрасте не только натощак, но и постпрандиально в течение дня легло в основу многократных измерений сахара крови глюкометром на протяжении 2 нед. Группы были сформированы из числа тех же детей, которые были включены в первый и второй этапы

$\%$

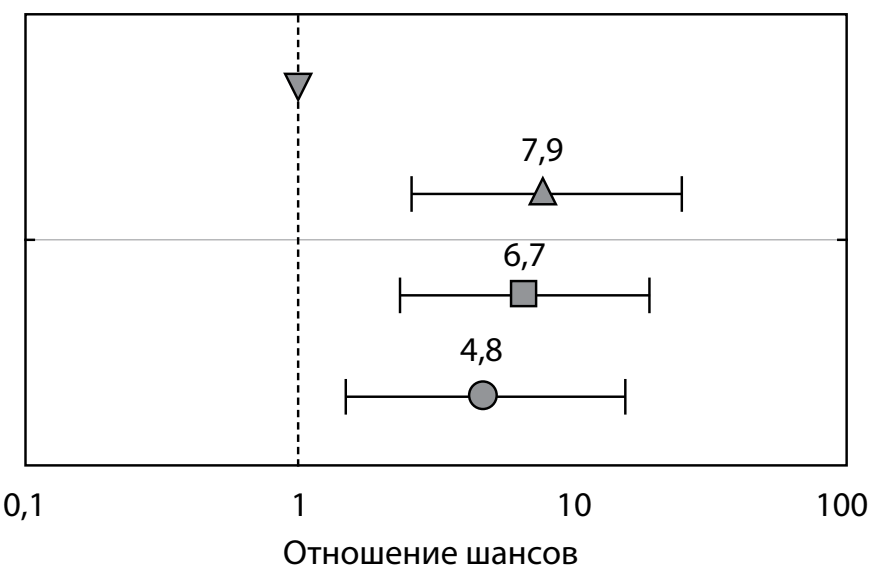

Группа $1 \square$ группа $2 \triangle$ Группа $3 \nabla$ группа 4

Рисунок 5. Риск рождения ребенка с неонатальной гипогликемией В зависимости от гликемии женщин во время беременности (статистический анализ проведен с использованием метода логистической регрессии). 
Таблица 3. Показатели уровня глюкозы капиллярной крови у детей раннего возраста (1-4 лет), зарегистрированные в течение 2 недель методом самоконтроля с использованием глюкометра

\begin{tabular}{cccc}
\hline Группа & $\begin{array}{c}\text { Гликемия натощак, } \\
\text { Me [min; max], ммоль/л }\end{array}$ & $\mathbf{p}^{*}$ & $\begin{array}{c}\text { Гликемия постпрандиальная, } \\
\text { Me [min; max], ммоль/л }\end{array}$ \\
\hline 1 & $4,7[4,05 ; 5,3]$ & & $5,68[5,2 ; 7,06]$ \\
2 & $4,78[4,41 ; 5,54]$ & 0,1 & $5,44[5,02 ; 5,67]$ \\
3 & $4,17[3,5 ; 5,11]$ & & $5,4[5,09 ; 5,89]$ \\
4 & $4,56[4,26 ; 5,17]$ & $5,15[4,56 ; 6,1]$ \\
\hline
\end{tabular}

Примечания: статистический анализ был проведен с использованием непараметрического критерия Краскела-Уоллиса. p $^{*}$ с сравнение гликемии натощак в четырех группах наблюдения. p** - сравнение постпрандиальной гликемии в четырех группах наблюдения.

исследования. Статистический анализ показателей глюкозы капиллярной крови не выявил различий в зависимости от принадлежности детей к группам наблюдения (табл. 3).

\section{Дополнительные результаты исследования}

В ходе анализа перинатального периода дана характеристика терапии ГСД у женщин, выполнена оценка хронических соматических заболеваний женщин, течения беременности и интранатального периода в зависимости от наличия ГСД. В группе 1 инсулинотерапию получали $12 \%$ женщин $(n=6)$, в то время как $88 \%(n=44)$ придерживались диеты; в группе $2-26 \%$ женщин ( $\mathrm{n}=23)$ получали инсулинотерапию, 74\% женщин (n=67) - диетотерапию; в группе 3 инсулинотерапию получали 35\% женщин ( $\mathrm{n=16})$, диетотерапию - 65\% $(\mathrm{n}=30)$ женщин.

Отмечалась более высокая частота встречаемости ожирения у женщин в группе ГСД (группа ГСД - 27,9\%, группа без ГСД - 14,9\%; p=0,01). Подтвержденных особенностей течения беременности у женщин с ГСД по сравнению с женщинами группы контроля выявлено не было ( $>0,05)$. При сравнении течения интранатального периода в группах наблюдения были выявлены значимые различия повышения травматизма во время родов (разрыв шейки матки, разрыв промежности) в группе женщин с ГСД (группа ГСД - 38,7\%, группа без ГСД $12,3 \% ; p=0,0000005)$. Анализ течения интранатального и раннего неонатального периода с педиатрической позиции не выявил различий в группах наблюдения (табл. 4).

\section{ОБСУЖДЕНИЕ}

Резюме основного результата исследования

Результаты проведенной работы свидетельствуют о важности целевых значений гликемии у беременных женщин с подтвержденным диагнозом ГСД, которые имеют прогностическое значение в определении рисков развития ряда антропометрических отклонений у новорожденных и особенностей течения неонатального периода. Наиболее низкие и сопоставимые с группой контроля риски развития антропометрических показателей «выше среднего», а также риск развития неонатальной гипогликемии ассоциированы с гликемией женщин натощак <5,1 ммоль/л и через 1 ч после еды <7,0 ммоль/л. В возрасте до 2 лет не было получено достоверных данных об отклонениях в показателях физического развития и метаболических параметров детей в зависимости

Таблица 4. Течение интранатального и раннего неонатального периода в группе детей у женщин с/без гестационного сахарного диабета

\begin{tabular}{|c|c|c|c|}
\hline Показатель & $\begin{array}{c}\text { Группа ГСД, } \\
\text { n=186 (\%) }\end{array}$ & $\begin{array}{c}\text { Группа без ГСД, } \\
n=114(\%)\end{array}$ & $\mathbf{p}^{*}$ \\
\hline Вакуум-экстракция плода & $5(2,7 \%)$ & $3(2,6 \%)$ & 0,9 \\
\hline Перелом ключицы новорожденного & $5(1,7 \%)$ & $1(0,8 \%)$ & 0,7 \\
\hline Кефалогематома новорожденного & $8(4,3 \%)$ & $4(3,5 \%)$ & 0,9 \\
\hline Физиологическая желтуха новорожденного & $43(23,1 \%)$ & $25(21,9 \%)$ & 0,9 \\
\hline Гемолитическая болезнь новорожденного & $4(2,2 \%)$ & 0 & 0,2 \\
\hline Фототерапия & $29(15,6 \%)$ & $17(19,9 \%)$ & 0,9 \\
\hline Грудное вскармливание & $132(70,8 \%)$ & $92(80,7 \%)$ & 0,08 \\
\hline Искусственное вскармливание & $3(1,6 \%)$ & $2(1,7 \%)$ & 0,9 \\
\hline Смешанное вскармливание & $51(27,6 \%)$ & $20(17,6 \%)$ & 0,1 \\
\hline Проведение инфузионной терапии растворами глюкозы & $12(6,5 \%)$ & $3(2,6 \%)$ & 0,2 \\
\hline Антибактериальная терапия & $5(2,7 \%)$ & $8(7,1 \%)$ & 0,08 \\
\hline Проведение нейросонографии & $31(16,7 \%)$ & $29(25,4 \%)$ & 0,07 \\
\hline Консультация невролога & $14(7,5 \%)$ & $15(13,2 \%)$ & 0,2 \\
\hline
\end{tabular}

Примечание: * - для статистического анализа использован критерий X2 для качественных признаков. 
от наличия ГСД у женщин. В возрасте детей до 4 лет не выявлено влияния уровня гликемии женщин с ГСД на значения глюкозы крови натощак и постпрандиально при мониторировании глюкометром на протяжении 14 дней. Полученные данные свидетельствуют о прогностической значимости уровня глюкозы крови во время беременности.

\section{Обсуждение основного результата исследования}

В настоящей публикации представлены некоторые данные исследования, посвященного актуальному вопросу современной перинатологии и педиатрии - изучению вклада метаболических факторов, действующих во время беременности, в процессы фетального программирования соматического здоровья детей в разные возрастные периоды. Учитывая продолжающиеся дебаты относительно влияния оптимальной гликемии во время беременности, ассоциированной с наименьшими негативными следствиями, на развитие плода и будущего ребенка, в нашем исследовании проведен сравнительный анализ параметров физического развития и углеводного обмена детей в зависимости от контроля материнской гликемии. Согласно данным литературы, подавляющее число исследований посвящено влиянию ГСД матери на течение перинатального периода или отдаленным воздействиям на здоровье детей вне зависимости от уровня гликемии во время беременности. Так, по результатам ранее проведенного исследования, у детей из группы ГСД чаще отмечалось развитие таких состояний, как дистоция плечиков, в 19\% случаев приводящая к перелому ключицы, в 7\% случаев - паралич Эрба и тяжелая асфиксия - у 1,4-5,3\% детей [8]. По результатам проведенного нами исследования подобных данных получено не было, и родовой травматизм был сопоставим в группе ГСД и группе контроля. Неоднократно была описана макросомия плода, характерная для прегестационных, а также моногенных форм диабета, манифестирующих во время беременности, как наиболее выраженное антропометрическое и морфофункциональное отклонение у детей $[9,10]$. В проведенном нами исследовании количество детей с макросомией было сопоставимо в обследуемых группах (группа ГСД 13,4\%, группа контроля 7,9\%; p=0,2), что свидетельствовало об отсутствии ассоциации данного признака в группе новорожденных у женщин с ГСД. При более подробном анализе антропометрических признаков новорожденных в группе ГСД была выявлена большая частота встречаемости показателей массы, отношения масса/длина тела и окружности головы «выше среднего» по сравнению с группой контроля. Наименьший и сопоставимый с группой контроля риск развития данных показателей был ассоциирован с наиболее низкой гликемией женщин во время беременности (натощак <5,1 ммоль/л и через 1 ч после приема пищи $<7,0$ ммоль/л), в то время как более высокие показатели гликемии повышали данный риск.

Одним из патогенетических звеньев неонатальной гипогликемии при ГСД является развитие гиперинсулинемии плода в ответ на персистирующую гипергликемию матери во время беременности $[11,12]$. После рождения повышенный уровень неонатального инсулина сохраняется и может привести к гипогликемии и подавлению механизмов метаболической компенсации. Младенцы, подвергшиеся гипогликемии в неонатальном периоде, демонстрируют отклонения на МРТ головного мозга и имеют риск задержки развития в более старшем возрасте, даже в случае легкой преходящей гипогликемии [13]. По результатам проведенного нами исследования, наиболее значимые отклонения углеводного обмена наблюдались в раннем неонатальном периоде, были представлены неонатальной гипогликемией, риск которой был наименьшим в группе наиболее низких значений глюкозы крови беременных женщин. Работы зарубежных авторов подтверждают важность уровня гликемии при ГСД и его ассоциацию с развитием неонатальной гипогликемии вне зависимости от терапии женщин (диета и/или инсулинотерапия) во время беременности [14], что также было продемонстрировано в проведенном нами исследовании.

В последние годы все больше внимания уделяется нескольким группам патологий детей, рожденных у женщин с ГСД, - избыток веса и ожирение, нарушения углеводного и липидного обмена, артериальная гипертензия. Однако полученные результаты не всегда однозначны и в большинстве случаев противоречивы. По данным одних авторов, была установлена ассоциация ГСД, увеличения массы тела и частоты ожирения у детей в течение первых 10 лет жизни [15], в то время как в другом исследовании данных ассоциаций не прослеживалось [16]. Проведенная нами работа позволила оценить и сравнить антропометрические показатели детей до возраста 2 лет. По ее результатам достоверных различий между детьми группы ГСД и группы контроля получено не было. Одно из наиболее масштабных исследований, посвященных изучению здоровья детей, рожденных у женщин с ГСД, под названием HAPO Follow-up Study, выявило независимые ассоциации ГСД с нецелевым уровнем гликемии с наличием инсулинорезистентности, нарушением толерантности к углеводам и риском развития ожирения у детей по сравнению с контрольной группой в возрасте 11-14 лет [17]. В то время как гипергликемия при нелеченых легких формах ГСД не приводила к повышению индекса массы тела, уровня гликемии натощак и инсулинорезистентности у детей в возрасте 5-10 лет по результатам исследований других авторов [18]. Проведенная нами работа позволила оценить некоторые метаболические параметры у детей более раннего возраста $(6,12$ и 24 мес). Так, при разовых измерениях глюкозы, инсулина, индекса HOMA-IR, холестерина и триглицеридов различий между группой ГСД и группой контроля выявлено не было. При дальнейшем наблюдении в возрасте детей от 1 до 4 лет при мониторировании гликемии натощак и постпрандиально на протяжении 14 дней значимых различий в группе ГСД и группе контроля также получено не было.

В целом полученные нами результаты показали как ассоциации более высоких трендов гликемии при ГСД с негативными следствиями для ребенка, так и возможность превенции последних при условии установления метаболического контроля гликемии во время беременности путем дискретного врачебного наблюдения и мотивации женщины на выполнение данных рекомендаций. 


\section{ЗАКЛЮЧЕНИЕ}

В проведенном нами исследовании основные отклонения в физическом развитии и метаболических параметрах детей, рожденных у женщин с ГСД, относились к раннему неонатальному периоду. Наименьшие риски отклонений антропометрических показателей и развития неонатальной гипогликемии были ассоциированы с уровнем гликемии женщин во время беременности натощак <5,1 ммоль/л и <7,0 ммоль/л через 1 ч после еды. По мере взросления детей отклонения нивелировались, и к 2 годам антропометрические и метаболические показатели в группах сравнения не различались. При многократном измерении глюкозы с помощью глюкометра натощак и постпрандиально в возрасте до 4 лет у детей разных групп наблюдения также не было выявлено различий. При анализе полученных данных сделано заключение о важности целевых значений гликемии во время беременности, так как они являются предикторами не только исходов беременности, но и развития отклоняющихся от нормы изменений со стороны здоровья новорожденных.

Следует подчеркнуть, что период катамнестического наблюдения за физическим развитием и метаболическим статусом детей был недостаточно продолжитель- ным и относился к периоду раннего возраста, в связи с чем требуется дальнейшее длительное наблюдение данной группы на протяжении всего периода детского возраста с регулярной, не реже одного раза в год, оценкой совокупности антропометрических и метаболических параметров, на основании которых можно будет сделать окончательное заключение об особенностях роста и развития в ассоциации с ГСД и уровнем гликемии во время беременности.

\section{ДОПОЛНИТЕЛЬНАЯ ИНФОРМАЦИЯ}

Финансирование работы. Исследование выполнено в структуре инициативной темы по плану научно-исследовательской работы НИЛ детской эндокринологии «НМИЦ им. В.А. Алмазова».

Конфликт интересов. Авторы декларируют отсутствие явных и потенциальных конфликтов интересов, связанных с публикацией настоящей статьи.

Участие авторов. Дейнега (Масель) А.С. - набор материала, анализ и статистическая обработка полученных данных, написание текста; Лискина А.С. - набор материала, анализ полученных данных; Алпысова С.А. - набор материала и анализ полученных данных третьего этапа исследования; Никитина И.Л. - концепция и дизайн исследования, редактирование, финальное утверждение рукописи.

\section{СПИСОК ЛИТЕРАТУРЫ | REFERENCES}

1. Barker DJP. In utero programming of chronic disease. Clin Sci. 1998;95(2):115-128. doi: https://doi.org/10.1042/cs0950115

2. Hod M, Kapur A, Sacks DA, et al. The International Federation of Gynecology and Obstetrics (FIGO) Initiative on gestational diabetes mellitus: A pragmatic guide for diagnosis, management, and care. Int J Gynecol Obstet. 2015;131:S173-S211. doi: https://doi.org/10.1016/S0020-7292(15)30033-3

3. International Diabetes Federation. IDF Diabetes Atlas. 8th ed. Brussels: IDF; 2017

4. Дедов И.И., Краснопольский В.И., Сухих Г.Т. Российский национальный консенсус «Гестационный сахарный диабет: диагностика, лечение, послеродовое наблюдение // Сахарный диабет. - 2012. - № 4. - C. 4-10. [Dedov II, Krasnopol'skiy VI, Sykhih GT. Russian National Consensus Statement on gestational diabetes: diagnostics, treatment and postnatal care. Diabetes Mellitus. 2012;4:4-10. (In Russ.)]. doi: https://doi.org/10.14341/2072-0351-5531

5. Blumer I, Hadar E, Hadden DR, et al. Introduction: Standards of Medical Care in Diabetes — 2019. Diabetes Care. 2019;42(S1):S1-S2. doi: https://doi.org/10.2337/dc19-Sint01

6. Blumer I, Hadar E, Hadden DR, et al. Diabetes and Pregnancy: An Endocrine Society Clinical Practice Guideline. J Clin Endocrinol Metab. 2013;98(11):4227-4249. doi: https://doi.org/10.1210/jc.2013-2465

7. Васюкова О.В., Витебская А.В. Инсулинорезистентность при ожирении у детей: спорность оценки // Проблемы эндокринологии. - 2009. - Т. 55. - №3. - C. 8-12. [Vasyukova OV, Vitebskaya AV. Insulin resistance in obese children: debate on assessment. Problems of Endocrinology. 2009;55(3):8-12. (In Russ.)]. doi: https://doi.org/10.14341/probl20095538-12

8. Евсюкова И.И., Кошелева Н.Г. Сахарный диабет: беременные и новорожденные. - М.: Миклош; 2009. [Evsyukova II, Kosheleva NG. Saharnyj diabet: beremennye i novorozhdennye. Moscow: Miklosh; 2009. (In Russ.)].

9. Kc K, Shakya S, Zhang H. Gestational diabetes mellitus and macrosomia: a literature review. Ann Nutr Metab. 2015;66(S2):14-20. doi: https://doi.org/10.1159/000371628

10. Зубкова Н.А., Бурумкулова Ф.Ф., Петрухин В.А., и др. Весоростовые показатели детей, рожденных от матерей с гестационным сахарным диабетом, обусловленным мутациями в гене глюкокиназы // Сахарный диабет. 2018. - T. 21. — №2. - C. 92-98. [Zubkova NA, Burumkulova FF, Petrukhin VA, et al. Birth weight and length in offsprings of mothers with gestational diabetes mellitus due to mutations in GCK gene. Diabetes Mellitus. 2018;21 (2):92-98. (In Russ.)]. doi: https://doi.org/10.14341/DM9429

11. Desoye G, Nolan CJ. The fetal glucose steal: an underappreciated phenomenon in diabetic pregnancy. Diabetologia. 2016;59(6):1089-1094. doi: https://doi.org/10.1007/s00125-016-3931-6

12. Pedersen J. Diabetes and pregnancy; blood sugar of newborn infants during fasting and glucose administration. Ugeskr Laeger. 1952;114(21):685.

13. McKinlay CJD, Alsweiler JM, Ansell JM, et al. Neonatal Glycemia and Neurodevelopmental Outcomes at 2 Years. N Engl J Med. 2015;373(16):1507-1518. doi: https://doi.org/10.1056/NEJMoa1504909

14. Voormolen DN, de Wit L, van Rijn BB, et al. Neonatal Hypoglycemia Following Diet-Controlled and Insulin-Treated Gestational Diabetes Mellitus. Diabetes Care. 2018;41(7):1385-1390. doi: https://doi.org/10.2337/dc18-0048

15. Hillier TA, Pedula KL, Vesco KK, et al. Impact of maternal glucose and gestational weight gain on child obesity over the first decade of life in normal birth weight infants. Matern Child Health J. 2016;20(8):1559-1568. doi: https://doi.org/10.1007/s10995-016-1955-7

16. Thaware PK, McKenna S, Patterson CC, et al. Unrelated mild hyperglycemia during pregnancy and anthropometric measures of obesity in offspring at age 5-7 years. Diabetes Care. 2015;38(9):1701-1706. doi: https://doi.org/10.2337/dc14-2797

17. Lowe WL, Scholtens DM, Kuang A, et al. Hyperglycemia and Adverse Pregnancy Outcome Follow-up Study (HAPO FUS): Maternal gestational diabetes mellitus and childhood glucose metabolism. Diabetes Care. 2019;42(3):372-380. doi: https://doi.org/10.2337/dc18-1646

18. Landon MB, Mele L, Varner MW, et al. The relationship of maternal glycemia to childhood obesity and metabolic dysfunction. J Matern Fetal Neonatal Med. 2020;33(1):33-41. doi: https://doi.org/10.1080/14767058.2018.1484094. 


\section{ИНФОРМАЦИЯ ОБ АВТОРАХ [AUTHORS INFO]}

*Дейнега (Масель) Алиса Сергеевна [Alisa S. Deynega (Masel)]; адрес: Россия, 197341, Санкт-Петербург, Коломяжский пр., д. 21, корп 2. [11/2 Kolomyazhskiy prospect, 197341 Saint-Petersburg, Russia]; ORCID: https://orcid.org/0000-0001-6314-0471; eLibrary SPIN: 8772-7809; e-mail: masel.alisa@gmail.com

Лискина Анастасия Сергеевна [Anastasiya S. Liskina]; ORCID: https://orcid.org/0000-0003-0307-5553; eLibrary SPIN: 5100-8480; e-mail: anastoz@mil.ru

Валиева Салимет Альбертовна [Salimet A. Valieva]; ORCID https://orcid.org/0000-0001-8314-9377; eLibrary SPIN: 3362-5058; e-mail: salimetalpysova@mail.ru

Никитина Ирина Леоровна, д.м.н. [Irina L. Nikitina, MD, PhD]; ORCID: https://orcid.org/0000-0003-4013-0785; eLibrary SPIN: 7707-4939; e-mail: nikitina0901@gmail.com

\section{ЦИТИРОВАТЬ:}

Дейнега (Масель) А.С., Лискина А.С., Валиева С.А., Никитина И.Л. Оценка физического развития и метаболического статуса детей раннего возраста, рожденных у женщин с гестационным сахарным диабетом // Сахарный диабет. 2021. — T. 24. — №4. - C. 325-333. doi: https://doi.org/10.14341/DM12463

\section{TO CITE THIS ARTICLE:}

Deynega (Masel) AS, Liskina AS, Valieva SA, Nikitina IL. Assessment of the physical development and metabolic status of children born to women with gestational diabetes. Diabetes Mellitus. 2021;24(4):325-333. doi: https://doi.org/10.14341/DM12463 